\title{
Two Fundamental Theorems on Deformations of Polarized Varieties
}

\section{Citation}

Matsusaka, T., and David Bryant Mumford. 1964. Two fundamental theorems on deformations of polarized varieties. American Journal of Mathematics 86(3): 668-684.

\section{Published Version}

doi: $10.2307 / 2373030$

\section{Permanent link}

http://nrs.harvard.edu/urn-3:HUL.InstRepos:3450065

\section{Terms of Use}

This article was downloaded from Harvard University's DASH repository, and is made available under the terms and conditions applicable to Other Posted Material, as set forth at http:// nrs.harvard.edu/urn-3:HUL.InstRepos:dash.current.terms-of-use\#LAA

\section{Share Your Story}

The Harvard community has made this article openly available.

Please share how this access benefits you. Submit a story.

Accessibility 


\title{
Two Fundamental Theorems on Deformations of Polarized Varieties
}

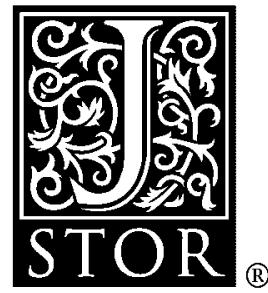

\author{
T. Matsusaka; D. Mumford
}

American Journal of Mathematics, Vol. 86, No. 3 (Jul., 1964), 668-684.

Stable URL:

http://links.jstor.org/sici?sici=0002-9327\%28196407\%2986\%3A3\%3C668\%3ATFTODO\%3E2.0.CO\%3B2-W

American Journal of Mathematics is currently published by The Johns Hopkins University Press.

Your use of the JSTOR archive indicates your acceptance of JSTOR's Terms and Conditions of Use, available at http://www.jstor.org/about/terms.html. JSTOR's Terms and Conditions of Use provides, in part, that unless you have obtained prior permission, you may not download an entire issue of a journal or multiple copies of articles, and you may use content in the JSTOR archive only for your personal, non-commercial use.

Please contact the publisher regarding any further use of this work. Publisher contact information may be obtained at http://www.jstor.org/journals/jhup.html.

Each copy of any part of a JSTOR transmission must contain the same copyright notice that appears on the screen or printed page of such transmission.

JSTOR is an independent not-for-profit organization dedicated to creating and preserving a digital archive of scholarly journals. For more information regarding JSTOR, please contact support@jstor.org. 


\title{
TWO FUNDAMENTAL THEOREMS ON DEFORMATIONS OF POLARIZED VARIETIES.
}

\author{
By T. Matsusaka and D. Mumford. ${ }^{1}$
}

Introduction. In contrast to the theory of moduli of curves, the global theory of moduli of higher dimensional varieties-with the exception of Abelian varieties-is largely unexplored. The work of the authors and of others ${ }^{2}$ has begun at least to clarify the problem, and to pose some plausible conjectures. One thing that is clear, however, is that there is a complexity here of a higher order of magnitude from that encountered for curves. The purpose of the present article is to present two results of a qualitative nature that limit the degree of possible complexity of various sought for varieties or scheme of moduli. The first result of ours asserts that two non-singular projectire varieties with polarizations, which are isomorphic as polarized varieties, remain isomorphic after specializations over a discrete valuationring, whenever they remain non-singular polarized varieties and at least one of them is non-ruled (cf. Th. 2). The second asserts that a set of nonsingular polarized surfaces, which are deformations of each other, can be realized as an algebraic family (i.e. a finite union of an irreducible algebraic family) of non-singular projective surfaces in a projective space, if their ranks are bounded; and, in fact, the set of non-singular surfaces with nondegenerate divisors with a given Hilbert polynomial and of any characteristic can be realized as an algebraic family over the ring of integers. From this, it can be shown that the variety of moduli of such surfaces, which are not ruled, is a finite union of $Q$-varieties, which will be disccissed in a near future.

In Chapter I, we shall settle the first result we mentioned. In Chapter II, we give an estimation for $l(X)$ when $X$ is a non-degenerate divisor on a projective variety. Our second main theorem will be settled in Chapter III, as well as in Chapter IV, under slightly different technique. In the first three Chapters, essentially the terminonolgy and conventions of Weil's book [18] are followed. In Chapter IV, because of the nature of the technique which

Received October 25, 1963.

${ }^{1}$ This work was done while the first named author was supported by the N.S.F. and the second named author was supported by the Sloan Foundation, and the Army Research Office (Durham).

${ }^{2}$ Cf. [5], [6], [8], [9], [10], [11]. 
are followed, essentially Grothendieck's terminology and conventions in [2] are followed. However, in order to keep the uniformity, the word "ample" (resp. "non-degenerate") is used for "very ample" (resp. "ample") in the sense of Grothendieck.

By a specialization of a variety or a cycle, we understand a reduction of such over a discrete valuation-ring (cf. [17]). For the theorem of RiemannRoch in general, we follow quite often the sheaf-theoretic terminology which can be found in [15] and [22]. Let $V$ be a normal variety and $M$ a finitely generated module of functions on $V$. When $Y=\inf _{g \in M}(\operatorname{div}(g))$, the set $\Lambda(M)$ of $V$-divisors $\operatorname{div}(g)-Y, g \in \mathbb{M}$, is called the reduced linear system determined by $M$. When $F$ is any positive $V$-divisor, $\Lambda(M)+F$ is called a linear system. Assume that $V$ is complete. When $X$ is a $V$-divisor, the set $L(X)$ of functions $g$ on $V$ such that $\operatorname{div}(g)+X \succ 0$ forms a finite dimensional vector space ([18], App. 1, Th. 3). We denote by $\Lambda(X)$ the set of positive $V$-divisors which are linearly equivalent to $X$, and call it the complete linear system determined by $X$. We denote by $|X|$ the support of $X$. We have $\Lambda(X)=\Lambda(L(X))+F$, where $F=X+\inf _{\mathcal{F} \in L(X)}(\operatorname{div}(g))$. We denote by $l(X)$ the dimension of $L(X)$. When $V$ is a projective variety, we denote by $o_{V}$ the sheaf of local rings on $V$, the defining sheaf of functions on a scheme $V$. If $X$ is a Cartier divisor on $V$, we denote by $\mathfrak{L}(X)$ the corresponding invertible sheaf. With this sheaf theoretic notations, $H^{0}(V, \mathfrak{L}(X))=L(X)$ when $V$ is normal. Moreover, when $V$ is a non-singular projective surface, $H^{2}(V, \mathfrak{L}(X))$ is isomorphic to the dual of $H^{0}(V, \mathfrak{L}(K(V)-X))$ and $\operatorname{dim} H^{1}(V, \mathfrak{R}(X))=s(X)$ is the superabundance of $X$. When there is no danger of confusion, we write $H^{i}(\mathfrak{L}(X))$ for $H^{i}(V, \mathfrak{\Omega}(X))$.

\section{Chapter I.}

THeorem $1 .^{3}$ Let $V$ be a complete abstract variety, $W$ an abstract variety and $T$ a birational correspondence between $V$ and $W$. Let $\mathfrak{o}$ be a discrete valuation-ring with the quotient field $k$, such that $V, W$ and $T$ are defined over $k$. Let $\left(V^{\prime}, W^{\prime}, T^{\prime}\right)$ be a specialization of $(V, W, T)$ over o and assume that $V^{\prime}, W^{\prime}$ are abstract varieties and that $V^{\prime}$ is complete. When $W^{\prime}$ is not a ruled variety, there is a component $T^{\prime \prime}$ of $T^{\prime}$ with the coefficient 1 in $T^{\prime}$ such that $T^{\prime \prime}$ is a birational correspondence between $V^{\prime}$ and $W^{\prime}$ and that $\operatorname{pr}_{i}\left(T^{\prime}-T^{\prime \prime}\right)=0$ for $i=1,2$.

Proof. From the compatibility of specializations with the operation of algebraic projection (cf. [17]), we see that $T^{\prime}$ has a component $T^{\prime \prime}$ with the

\footnotetext{
${ }^{3}$ 'I his theorem was pionted out to us by M. Artin.
} 
following properties: (a) $\operatorname{pr}_{2} T^{\prime \prime}=W^{\prime}$; (b) the coefficient of $T^{\prime \prime}$ in $T^{\prime}$ is 1 ; (c) $\operatorname{pr}_{2}\left(T^{\prime}-T^{\prime \prime}\right)=0$. Let $\mathfrak{p}$ be the maximal ideal of $\mathfrak{o}$ and $\kappa$ the residue field of $\mathfrak{o}$ with respect to $\mathfrak{p}$. Let $\left(x^{\prime}\right)$ be a generic point of a representative of $W^{\prime}$ over $\kappa$. Then, there is a representative $(x)$ of a generic point of $W$ over $k$ such that $\left(x^{\prime}\right)$ is a specialization of $(x)$ over $\mathfrak{v}$, over

$$
(V, W, T) \stackrel{\mathfrak{o}}{\longrightarrow}\left(V^{\prime}, W^{\prime}, T^{\prime}\right)
$$

(cf. [17], Th. 7 ). Let $R_{v}$ be the specialization-ring of the specialization $(x) \stackrel{\mathfrak{o}}{\longrightarrow}\left(x^{\prime}\right)$ in $k(x)$. Then $R_{v}$ is a discrete valuation ring of $k(x)$ (cf. [1\%], Prop. 5 and Th. 15). Hence, it determines a valuation $v$ of $k(x)$. Let $Q \times(x)$ be a generic point of $T$ over $k$. Since $V$ and $V^{\prime}$ are complete, there is at least one representative $(y)$ of $Q$ such that the coordinates $y_{i}$ of (y) are in $R_{v}$, that $\left(y^{\prime}\right)$ is a representative of $Q^{\prime}$ if $(Q \times(x),(y))$ $\mathfrak{D}$ $\left.\left(Q^{\prime} \times\left(x^{\prime}\right), y^{\prime}\right)\right)$ and that $Q^{\prime} \times\left(x^{\prime}\right)$ is contained in $\left|T^{\prime}\right|$. When that is so, $Q^{\prime} \times\left(x^{\prime}\right)$ is contained in $T^{\prime \prime}$; in fact, it is a generic point of $T^{\prime \prime}$ since $\operatorname{pr}_{2}: T^{\prime \prime} \rightarrow W^{\prime}$ is birational and $\left(x^{\prime}\right)$ is a generic point of $W^{\prime}$ over $\bar{\kappa}$. It follows that $Q^{\prime}$ is a generic point of the projection $A$ of $T^{\prime \prime}$ on $V^{\prime}$ over $\bar{\kappa}$. Let $R$ be the specialization ring of $(y) \stackrel{\mathfrak{o}}{\longrightarrow}\left(y^{\prime}\right)$. Then, the valuation $v$ is a prime divisor of $R$ in the sense of Abhyankar, and $W^{\prime}$ is a ruled variety over $A$ unless $A=V^{\prime}$ (cf. [1], Prop. 3). Therefore, $A=V^{\prime}$. When that is so, $T^{\prime \prime}$ is a birational correspondence between $V^{\prime}$ and $W^{\prime}$, which can be seen easily, using the compatibility of specializations with the operation of intersectionproduct (cf. [17]).

THeOREM 2. Let $\mathfrak{o}$ be a discrete valuation-ring with the quotient field $k$; let $V$ and $W$ be non-singular projective varieties, defined over $k$, and $T$ the graph of an isomorphism, defined over $k$, between $V$ and $W$. Let $X$ (resp. $Y$ ) be a non-degenerate divisor on $V$ (resp. W), both rational over $k$, such that $Y=T(X)$. Let $(V, W, X, Y, T) \stackrel{\mathfrak{o}}{\longrightarrow}\left(V^{\prime}, W^{\prime}, X^{\prime}, Y^{\prime}, T^{\prime}\right)$ and assume that $V^{\prime}, W^{\prime}$ are non-singular and that $X^{\prime}\left(\right.$ resp. $\left.Y^{\prime}\right)$ is also non-degenerate on $V^{\prime}$ (resp. $\left.W^{\prime}\right)$. Then $T^{\prime}$ is the graph of an isomorphism between $V^{\prime}$ and $W^{\prime}$, if one of the $V^{\prime}, W^{\prime}$ is not ruled.

Proof. By Theorem 1, we have $T^{\prime \prime}=T^{\prime \prime}+T^{*}$, where $T^{\prime \prime}$ is a birational correspondence between $V^{\prime}$ and $W^{\prime}$, and $\operatorname{pr}_{l} T^{*}=0$ for $l=1$, 2. Let $F_{1}, \cdots, F_{t}$ be the projections of the components of $T^{*}$ on $V^{\prime}$. Note that none of the $F_{i}$ is 0-dimensional: for if $F_{i}$ were 0-dimensional, the corresponding component 
of the $n$-dimensional cycle $T^{*}$ would have to be of the form $F_{i} \times W^{\prime}$, and this contradicts $\operatorname{pr}_{2} T^{*}=0$. If $X^{\prime}{ }_{m}$ is a divisor in $\Lambda\left(m X^{\prime}\right)$, then $T^{\prime}$ and $X^{\prime}{ }_{m} \times W^{\prime}$ intersect properly if and only if $\left|X^{\prime}{ }_{m}\right| \unrhd F_{i}$ for any $i$. Let $U$ be the set of such divisors $X_{m}^{\prime}$. For every such $X_{m}^{\prime}, T^{\prime}\left(X_{m}^{\prime}\right)$ is defined. The Chowvariety of $U$, i. e. the set of Chow-points of members of $U$, is an open subset of that of $\Lambda\left(T^{\prime}\left(X_{m}^{\prime}\right)\right)$. When $U$ is not empty, the mapping $X^{\prime}{ }_{m} \rightarrow T^{\prime}\left(X^{\prime}{ }_{m}\right)$ defines, as is well-known, an injection of $U$ into $\Lambda\left(T^{\prime}\left(X^{\prime}{ }_{m}\right)\right)$; and, as a matter of fact, defines an injective linear rational map of the Chow variety of $U$ into that of $\Lambda\left(T^{\prime}\left(X_{m}^{\prime}\right)\right)$ ef. [18], Chap. IX, Th. 3 and [18], Chap. VIII, Th. 4). Now assume that (a) $T^{\prime}\left(X_{m}^{\prime}\right) \sim m Y^{\prime}$ and (b) $l\left(m X^{\prime}\right)=l\left(m Y^{\prime}\right)$ for large $m$.

Suppose that $P^{\prime}$ is a point of $V^{\prime}$ and let $\Lambda\left(m X^{\prime}\right)_{P^{\prime}}$ be the linear subsystem of divisors which pass through $P^{\prime}$. For sufficiently large $m, m X^{\prime}$ is ample, hence $P^{\prime}$ is the only base point of $\Lambda\left(m X^{\prime}\right)_{P^{\prime}}$, hence $\Lambda\left(m X^{\prime}\right)_{P^{\prime}} \cap U$ is not empty. Then the set $A$ of divisors $T^{\prime}\left(Z^{\prime}\right), Z^{\prime} \in \Lambda\left(m X^{\prime}\right)_{P^{\prime}} \cap U$, consists of divisors passing through every point $Q^{\prime}$ such that $P^{\prime} \times Q^{\prime} \in\left|T^{\prime}\right|$. If there were more than one such $Q^{\prime}$, the closure of the Chow-variety of $A$ is at least of co-dimension 2 in that of $\Lambda\left(m Y^{\prime}\right)$, since $m Y^{\prime}$ is ample for sufficiently large $m$. On the other hand, its co-dimension has to be 1 as the closure of the image of the Chow-variety of $\Lambda\left(m X^{\prime}\right)_{P^{\prime}} \cap U$ by the injective rational map, since $l\left(m X^{\prime}\right)=l\left(m Y^{\prime}\right)$. Hence $T^{*}=0$ and $T^{\prime}$ is single-valued on the points of $V^{\prime}$, hence everywhere regular by Zariski's Main Theorem. Similarly $T^{\prime-1}$ is everywhere regular.

To prove (a) and (b), note that $p_{a}(m X)=p_{a}(m Y)$ for all integers $m$. Hence $p_{a}\left(V^{\prime}\right)=p_{a}(V)=p_{a}(W)=p_{a}\left(W^{\prime}\right)$; and $p_{a}\left(m X^{\prime}\right)=p_{a}(m X)=p_{a}(m Y)$ $=p_{a}\left(m Y^{\prime}\right)$ for all integers $m$ (cf. [13]). It follows that $l(m X)=l(m Y)$ $=l\left(m X^{\prime}\right)=l\left(m Y^{\prime}\right)$ for large positive integer $m$ by the theorem of RiemannRoch (cf. [21]). Thus (b) is satisfied. Now let $C$ and $D$ be the supports of the Chow-varieties of $\Lambda(m X), \Lambda(m Y)$. Since the linear equivalence is preserved under specializations (cf. [1\%]), $C^{\prime}, D^{\prime}$ will be the supports of the Chow-varieties of $\Lambda\left(m X^{\prime}\right), \Lambda\left(m Y^{\prime}\right)$ for large $m$, if $(C, D) \stackrel{\mathfrak{o}}{\longrightarrow}\left(C^{\prime}, D^{\prime}\right)$. Then (a) follows from the compatibility of specializations with the operation of intersection-product and from the invariance of linear equivalence under specializations.

Let $V$ and $V^{\prime}$ be two complete non-singular polarized varieties (cf. [20]), $k$ a field of definition of $V$ and $\mathfrak{o}$ a discrete valuation ring with the quotient field $k$. Let $X$ be a polar divisor of $W$ and $W, W^{\prime}$ the underlying varieties of $V, V^{\prime}$. If $(W, X) \stackrel{\mathfrak{o}}{\longrightarrow}\left(W^{\prime}, X^{\prime}\right)$ and $X^{\prime}$ is a polar divisor of $V^{\prime}$, we 
shall say that $V^{\prime}$ is a specialization of $V$ over $\mathfrak{o}$. With this definition, de have the following corollary.

Corollary 1. Let $\boldsymbol{V}$ and $\boldsymbol{W}$ be two varieties over a discrete valuation ring $\mathfrak{o}$ (i.e. p-variety in the sense of Shimura; a scheme in the sense of Grothendieck). Let generic fibres $V, W$ of $\boldsymbol{V}, \boldsymbol{W}$ be non-singular projective varieties, defined over the quotient field $k$ of $\mathrm{o}$. Let the special fibres $V^{\prime}, W^{\prime}$ be non-singular projective varieties. Assume that $V, W, V^{\prime}, W^{\prime}$ are underlying varieties of polarized varieties $\tilde{V}, \tilde{W}, \tilde{V^{\prime}}, \tilde{W}^{\prime}$ and that $(V, W) \stackrel{\mathfrak{o}}{\longrightarrow}\left(V^{\prime}, W^{\prime}\right)$ can be extended to $(\tilde{V}, \tilde{W}) \stackrel{\mathfrak{o}}{\longrightarrow}\left(\tilde{V}^{\prime}, \tilde{W}^{\prime}\right)$. Then, when there is an isomorphism $\tilde{f}$ between $\tilde{V}$ and $\tilde{W}$ over $k, \tilde{f}$ can be extended to an isomorphism $\boldsymbol{f}$ of $\boldsymbol{V}$ and $\boldsymbol{W}$, if $W^{\prime}$ is not ruled. Moreover, the graph of $\tilde{f}$ specializes to the graph of an isomorphism $f^{\prime}$ between $\tilde{V}^{\prime}$ and $\tilde{W}^{\prime}$ over $\mathfrak{o}$.

Corollary 2. Let $V$ be a projective, non-ruled, non-singular variety with a structure of polarization and $G$ the connected component, containing the identity, of the group of automorphisms of $V$. Then $G$ is an Abelian variety.

Proof. The group of automorphisms of $V$ is an algebraic group (cf. [8]). If $G$ is not complete, the graph of an automorphism, corresponding to a suitable element of $G$, can be specialized, over some field of definition of $V$, to a $V \times V$-cycle which is not the graph of an automorphism. This is impossible by Theorem 2. Hence $G$ is complete and is an Abelian variety by the theorem of Chevalley (cf. [19], Th. 5).

\section{Chapter II.}

Let $V^{n}$ be a normal projective variety and $X$ a non-degenerate divisor on $V . L(m X)$ defines a projective embedding $f_{m}$ of $V$ for large $m$ by the definition. Let $W^{r}$ be a simple subvariety of $V$ and $k$ a common field of definition for $W$ and $V$, over which $X$ is rational. Then $L(m X)$ has a basis over $k$ (cf. [18], Ch. IX, Cor. 1 of Th. 8). Let $A_{1}, \cdots, A_{r}$ be independent generic divisors of $\Lambda(m X)$ over $k$. Then every component of $W \cap A_{1} \cap \cdots \cap A_{r}$ is simple on $V$ and on $W$ (cf. [18], Ch. V, Th. 1). We set $\left[W \cdot X^{(r)}\right]=\left(1 / m^{r}\right) \operatorname{deg}\left(W \cdot A_{1} \cdots A_{r}\right)$ and $X^{(n)}=\left[V \cdot X^{(n)}\right]$. Then $\left[W \cdot X^{(r)}\right]$ does not depend upon the choice of independent generic divisors $A_{1}, \cdots, A_{r}$. Moreover, it does not depend upon the choice of $m$, as long as it is sufficiently large, and is a positive integer (cf. Bezout's theorem). 
THEOREM 3. Let $V^{n}$ be a normal projective variety and $X$ a nondegenerate divisor on $V$. Then $l(X) \leqq X^{(n)}+n$.

Proof. If $l(X) \leqq 1$, there is nothing to prove. If $n=1$, our theorem is an immediate consequence of the theorem of Riemann-Roch. Therefore, we assume that $l(X)>1$ and that $n>1$. Let $X_{0}$ be a generic divisor of the complete linear system $\Lambda(X)$. Then $X_{0}=\sum_{1}^{d} Y_{i}+F$, where $F$ is the fixed component of $\Lambda(X)$ and $Y_{i} \neq Y_{j}$ for $i \neq j$, since $\Lambda(X)$ is complete (cf. [18], Ch. IX, Cor. of Th. 15). If $d>1$, the $Y_{i}$ are generic divisors of one and the same pencil on $V$ by the theorem of Bertini (cf. [18], Ch. IX, Th. 17). Hence $\operatorname{dim} \Lambda(X)=l(X)-1 \leqq d$. On the other hand, we get $d \leqq X^{(n)}$ by computing $X^{(n)}=\left[\left(\sum_{1}^{d} Y_{i}+F\right) \cdot X^{(n-1)}\right]$. Therefore, our theorem is true in this case also.

Assume now that $d=1$. Then $X_{0}=Z+F$, where $Z$ is an absolutely irreducible subvariety of $V$. Let $K$ be an algebraically closed field, containing $k$, over which $Z$ and $F$ are rational, and $\left(Z^{*}, \alpha\right)$ a normalization of $Z$ over $K$. Let $m_{0}$ be a positive integer such that $m X$ is ample for $m \geqq m_{0}$ and $X_{m}$ a generic divisor of $\Lambda(m X)$ over $K$ for such $m$. We note that every component of $X_{m} \cap Z$ is simple both on $V$ and $Z$ and is proper on $V$. We contend that: (a) When $g \in L\left(X_{m+1}-X_{m}\right), g \rightarrow g^{*}=g \circ \alpha^{-1}$ is a homomorphism of $L\left(X_{m+1}-X_{m}\right)$ into $L\left(X^{*}\right)$, where $X^{*}=\alpha\left(Z \cdot\left(X_{m+1}-X_{m}\right)\right)$; (b) The kernel of the above homomorphism is a vector space of dimension 1 ; (c) $X^{*}$ is nondegenerate on $Z^{*}$ and $X^{*(n-1)} \leqq X^{(n)}$. Our theorem will be an immediate consequence of (a), (b), (c). For, we have $l\left(X^{*}\right) \leqq X^{*(n-1)}+(n-1)$ by the induction hypothesis, hence $l\left(X^{*}\right) \leqq X^{(n)}+(n-1)$ by $(\mathrm{c})$, and $l(X)=l\left(X_{m+1}-X_{m}\right) \leqq l\left(X^{*}\right)+1$ by $(\mathrm{a})$ and $(\mathrm{b})$.

To prove (a), we may assume that $g^{*} \neq 0$. We first remark the following two facts: (i) If $U$ is a subvariety of $Z$ of co-dimension 1 , which is simple both on $V$ and $Z$, and $g^{\prime}$ is the function induced on $Z$ by $g$, then the coefficient of $U$ in $\operatorname{div}(g) \cdot Z$, that of $U$ in $\operatorname{div}\left(g^{\prime}\right)$ and that of $\alpha(U)$ in $\operatorname{div}\left(g^{*}\right)$ all coincide; (ii) If $W^{*}$ is a component of $\operatorname{div}\left(g^{*}\right)_{\infty}=g^{*-1}(\infty)$, its geometric image $W$ by $\alpha^{-1}$ is a component of $X_{m+1} \cap Z$, and is simple both on $V$ and $Z$. In fact, $U$ has the same coefficient $a$ in $\operatorname{div}(g) \cdot \mathrm{Z}$ as in $\operatorname{div}\left(g^{\prime}\right)$ (cf. [18]-IX, Th. 3). Since $g^{*}$ can be written as $g^{\prime} \circ \alpha^{-1}$, and since $\alpha$ is biregular along $U$, it follows that the coefficient of $\alpha(U)$ in $\operatorname{div}\left(g^{*}\right)$ is also $a$. As for (ii), $g^{*}$ is not finite along $W^{*}$ (i. e. at a generic point of $W^{*}$ over a field of definition of $W^{*}$, containing $K$ ), and hence, $g$ is not also finite along $W$. Consequently, $W \subset\left|g^{-1}(\infty)\right|=\left|X_{m+1}\right|$ and $W$ is a component of $Z \cap X_{m+1}$. 
Now let $U$ be a component of $X_{m} \cap Z$ or of $X_{m+1} \cap Z$. Since

$$
\operatorname{div}(g) \cdot Z+Z \cdot\left(X_{m+1}-X_{m}\right) \succ 0,
$$

it follows that the coefficient of $\alpha(U)$ in $\operatorname{div}\left(g^{*}\right)+X^{*}$ is non-negative by (i). Therefore, if $\operatorname{div}\left(g^{*}\right)+X^{*}$ has a component $W^{*}$ of negative coeificient, it is a component of $\operatorname{div}\left(g^{*}\right)_{\infty}=g^{*-1}(\infty)$, which is impossible by (ii).

To prove (b), let $g$ be a function in $L\left(X_{m+1}-X_{m}\right)$ such that $g^{*}=0$. Then $\operatorname{div}(g)=W-\left(X_{m+1}-X_{m}\right)$, where $W$ is a positive $V$-divisor such that $Z$ is a component of it. Since $W \sim X_{m+1}-X_{m} \sim X$, it follows that $W=Z+F$ and that $g$ is uniquely determined up to a constant factor. (b) is thereby proved.

To prove (c), choose a positive integer $r \geqq m_{0}$ and identify $\Lambda(r X)$ with the linear system of hyperplane sections of $V$ by means of the embedding $f_{r}$. Let $s$ be another large positive integer. Then $s X_{r} \sim s r X_{m+1}-s r X_{m}$ and $s X_{r}$, $s r X_{m}, s r X_{m+1}$ are sections of $V$ by hypersurfaces of degrees $s, s m, s(m+1)$ respectively, since the linear system of hypersurface sections of a normal projective variety is complete when the degree of hypersurfaces is large enough (Zariski's normalization theorem). Consequently, $s X_{r} \cdot Z, s r X_{m} \cdot Z, s r X_{m+1} \cdot Z$ are also sections of $Z$ by hypersurfaces of degrees $s, s m, s(m+1)$. When $s$ is chosen large enough so that $\alpha$ is determined by homogeneous functions of homogenity $s, \alpha\left(s X_{r} \cdot Z\right), \alpha\left(s r X_{m} \cdot Z\right), \alpha\left(s r X_{m+1}\right)$ are hypersurface sections of $Z^{*}$ by hypersurfaces of degrees $1, m, m+1$ respectively. Hence

$$
\alpha\left(s X_{r} \cdot Z\right) \sim \operatorname{sr} \alpha\left(Z \cdot\left(X_{m+1}-X_{m}\right)\right) .
$$

Thus, $X^{*}=\alpha\left(Z \cdot\left(X_{m+1}-X_{m}\right)\right)$ is non-degenerate. $\Lambda(r X)$ is the linear system of hyperplane sections of $V$. Hence

$$
X^{(n)}=\left(1 / r^{n-1}\right) \operatorname{deg}(X)=\left(1 / r^{n-1}\right) \operatorname{deg}(Z+F) \geqq\left(1 / r^{n-1}\right) \operatorname{deg}(Z) .
$$

$\Lambda\left(s r X^{*}\right)$ is the linear system of hyperplane sections of $Z^{*}$. Hence $X^{*(n-1)}$ $=\left(1 /(s r)^{n-1}\right) \operatorname{deg}\left(Z^{*}\right)$. But $\operatorname{deg}\left(Z^{*}\right)=s^{n-1} \operatorname{deg}(Z)$ as is well-known and easy to see. (c) is thus proved.

Remark 1. Let $\mathfrak{\&}$ be a non-degenerate invertible sheaf (ample invertible sheaf in the sense of Grothendieck) on a projetive variety $V$. Let $d$ be the leading coefficient of $\chi\left(\Omega^{m}\right)$. Then it is easy to deduce that $\operatorname{dim} H^{0}(\Omega)$ $\leqq d+\operatorname{dim} V$ from our theorem. In fact, when $\left(V^{*}, \beta\right)$ is a normalization of $V$ and $X$ a Cartier divisor on $V^{*}$ determined by $\mathfrak{R}$, then $X$ is non-degenerate and $d=X^{(n)}$ if $\operatorname{dim} V^{*}=n$.

Remark 2. In our theorem, we assumed that $X$ is non-degenerate. Assume now that $V$ is a non-singular projective surface and $X$ a $V$-divisor 
such that $X^{(2)}>0$ and $[Y \cdot X]>0$ for all positive $V$-divisors $Y$. Then, we can prove directly that $l(X) \leqq X^{(2)}+2$. In fact, we may assume, as in the proof of our theorem, that a generic divisor $X_{0}$ of $\Lambda(X)$ is of the form $Z+F$, where $Z$ is an irreducible curve. Let $Y$ be a $V$-divisor such that $Y \sim X$ and that $|Y|$ contains neither $Z$ nor any singular point of $Z$. Then $[X \cdot Z]=[Y \cdot Z] \leqq X^{(2)}$ and $L(X)$ and $L(Y)$ are isomorphic. As in the proof of our theorem, $L(Y)$ induces on $Z$ a module $M^{\prime}$ of functions on $Z$; $\alpha^{-1}\left(M^{\prime}\right)$ is then a submodule of $M^{*}=L\left(\alpha^{-1}(Z \cdot Y)\right)$ and the kernel of the homomorphism $L(Y) \rightarrow M^{*}$ is a vector space of dimension 1 . Hence, we have our inequality by the theorem of Riemann-Roch. Our divisor $X$ is in fact a non-degenerate divisor on $V$ according to [12], and our theorem is available according to this. But using this remark and our Theorem 4, we recover this result.

\section{Chapter III.}

Let $V$ be a non-singular projective surface and $X, Y$ two divisors. There is a $V$-divisor $X^{\prime}$ such that $X^{\prime} \sim X$ and that $X^{\prime}$ and $Y$ intersect properly on $V$. We denote by $X_{\wedge} Y$ the intersection-product $X^{\prime} \cdot Y$, and by $[X \cdot Y]$ the degree of $X_{\wedge} Y$. When $X=Y,[X \cdot X]$ is denoted by $X^{(2)}$. We denote by $K(V)$ a canonical divisor on $V$ and set $p_{a}(X)=(1 / 2)[X \cdot(X+K(V))]+1$. When $X$ is irreducible, $(X+K(V))_{\wedge} X$ is a canonical divisor $K(X)$ of $X$ and $\operatorname{deg}(K(X))=2 p_{a}(X)-2$ (cf. [16]). When $X=\sum_{i} a_{i} X_{i}$, we have

$$
\begin{aligned}
p_{a}(X)=\sum_{i} a_{i} p_{a}\left(X_{i}\right) & +\sum_{i}(1 / 2) a_{i}\left(a_{i}-1\right) X_{i}^{(2)} \\
& +\sum_{i, j ; i \neq j}(1 / 2) a_{i} a_{j}\left[X_{i}, X_{j}\right]-\sum_{i} a_{i}-1 .
\end{aligned}
$$

According to the theorem of Riemann-Roch on $V$, we have

$$
l(X)-s(X)+l(K(V)-X)=X^{(2)}-p_{a}(X)+p_{a}(V)+2 .
$$

1. Denote by $\Sigma$ the set of pairs $(V, X)$ of a projective non-singular surface $V$ and a $V$-divisor $X$ satisfying the following conditions.

(I) $[X \cdot Y]>0$ whenever $Y$ is a positive $V$-divisor;

(II) $0<X^{(2)}<c_{1}$;

(III) $\left|p_{a}(X)\right|<c_{2}$;

(IV) $\left|p_{a}(V)\right|<c_{3}$.

In order to simplify further discussions, we assume that the constants $c_{i}$ $(i>3)$ which will be introduced are positive integers, satisfying $c_{i}>c_{i-1}$ and depending only upon $c_{1}, c_{2}, c_{3}$. 
LeMma 1. There are constants $c_{4}, c_{5}$ such that $|[X \cdot K(V)]|<c_{4}$ and that $p_{a}(m X)>0, l(K(V)-m X)=0, m^{2} X^{(2)}-p_{a}(m X)+p_{a}(V)+1>0$ whenever $(V, X) \in \Sigma$ and $m>c_{5}$.

This is an easy consequence of (I), (II), (III), (IV), the theorem of Riemann-Roch and of the formula (1).

Lemma 2. Let $(V, X)$ be a member of $\Sigma$ and $T=\sum_{1}^{t} a_{i} Y_{i}$ the reduced expression for a member $T$ of $\Lambda\left(2 c_{5} X\right)$. Then, there are constants $c_{6}, c_{7}, c_{8}$ and $c_{9}$ with the following properties:

(i) $\sum_{1}^{t} a_{i}<c_{6}$;

(ii) $\left|\left[Y_{i} \cdot Y_{j}\right]\right|<c_{7}$;

(iii) $\left|\left[K(V) \cdot Y_{i}\right]\right|<c_{8} ; 0 \leqq p_{a}\left(Y_{i}\right)<c_{8}$;

(iv) The multiplicity of any point on $Y_{i}$ is at most $c_{9}$.

Proof. (i) is a consequence of $\sum_{1}^{t} a_{i} \leqq \sum_{1}^{t} a_{i}\left[Y_{i} \cdot X\right]=[T \cdot X] \leqq 2 c_{1} c_{5}$. (ii) and (iii) follow from the three inequalities:

(A) $a_{i} Y_{i}^{(2)}+\sum_{i \neq j} a_{j}\left[Y_{i} \cdot Y_{j}\right]=\left[Y_{i} \cdot 2 c_{5} X\right] \leqq \sum a_{i}\left[Y_{i} \cdot 2 c_{5} X\right] \leqq 4 c_{5}{ }^{2} c_{1}$.

(B) $-2 \leqq 2 p_{a}\left(Y_{i}\right)-2=\left[Y_{i} \cdot\left(Y_{i}+K(V)\right)\right]$.

(C) $\sum a_{i}\left[K(V) \cdot Y_{i}\right] \leqq 2 c_{4} c_{5}$.

In fact, (A) gives an upper bound for every $Y_{i}{ }^{(2)}$. Hence, (B) gives a lower bound for every $\left[K(V) \cdot Y_{i}\right]$. Then $(\mathrm{C})$ gives an upper bound for every $\left[K(V) \cdot Y_{i}\right]$ and (iii) is proved. Returning to (B), we obtain a lower bound for every $Y_{i}^{(2)}$, and using this, (A) gives upper bounds for all $\left[Y_{i} \cdot Y_{j}\right]$. This gives (ii), since $\left[Y_{i} \cdot Y_{j}\right] \geqq 0$ if $i \neq j$. Finally, the arithmetic genus of $Y_{i}$ is bounded by (ii) and (iii). If the $r_{i j}$ are the multiplicities of the singular points $x_{i j}$ of $Y_{i}$, an inequality of Noether (cf. [4]) states

$$
\sum_{j} r_{i j}\left(r_{i j}+1\right) / 2+p_{a}\left(Y_{i}^{*}\right) \leqq p_{a}\left(Y_{i}\right)
$$

where $Y_{i}^{*}$ is a non-singular model of $Y_{i}$. This gives (iv).

The following lemma is an easy consequence of the generalized RiemannRoch theorem for curves.

Lemma 3. Let $W$ be a non-singular surface in a projective space and $Y$ a divisor on $W$. Let $C$ be an irreducible curve on $W$ such that $[Y \cdot C]$ $>2 p_{a}(C)-2$. Then $H^{1}(\mathfrak{L}(Y) / \mathfrak{L}(Y-C))=0$. 
Corollary. Using the same assumptions and notations of our lemma, $s(Y-C)=s(Y)$ if and only if

$$
0 \rightarrow H^{0}(\mathfrak{L}(Y-C)) \rightarrow H^{0}(\mathfrak{L}(Y)) \rightarrow H^{0}(\mathfrak{L}(Y) / \mathfrak{R}(Y-C)) \rightarrow 0 .
$$

Proof. This is an immediate consequence of our lemma and of an exact sequence $0 \rightarrow \mathfrak{L}(Y-C) \rightarrow \mathfrak{L}(Y) \rightarrow \mathfrak{L}(Y) / \mathfrak{R}(Y-C) \rightarrow 0$.

Lemma 4. Let $T=\sum_{i} a_{i} Y_{i}$ be a positive divisor on a non-singular projective variety $W, \Lambda(A)$ a complete linear system on $W$ and assume that $\left[\left(A-T^{\prime}\right) \cdot Y_{i}\right]>2 p_{a}\left(Y_{i}\right)-2$ for all $i$ and for all $T^{\prime}$ such that $0 \prec T^{\prime} \prec T$. Then we have $H^{1}(\mathfrak{R}(A) / \mathfrak{I}(A-T))=0$.

Proof. If $\sum_{i} a_{i}=1$, our lemma follows from Lemma 3. Assume that our lemma has been proved for those positive $W$-divisors $T^{\prime \prime}=\sum_{i} a^{\prime \prime}{ }_{i} Y_{i}$ with $\sum_{i} a^{\prime \prime}{ }_{i}<\sum_{i} a_{i}$. Set $T^{\prime}=\sum_{i} a_{i}^{\prime} Y_{i}$ with $a_{1}-1=a^{\prime}{ }_{1}, a_{i}=a_{i}^{\prime}$ for $i \geqq 2$. In the exact cohomology sequence of an exact sequence

$$
0 \rightarrow \mathfrak{L}\left(A-T^{\prime}\right) / \mathfrak{R}(A-T) \rightarrow \mathfrak{Z}(A) / \mathfrak{Z}(A-T) \rightarrow \mathfrak{R}(A) / \mathfrak{R}\left(A-T^{\prime}\right) \rightarrow 0,
$$

we have $H^{1}\left(\mathfrak{L}(A) / \mathfrak{L}\left(A-T^{\prime}\right)\right)=0$ by the induction assumption, and $H^{1}\left(\mathfrak{L}\left(A-T^{\prime}\right) / \mathfrak{R}(A-T)\right)=0$ by our assumption and Lemma 3 . Hence we get $H^{1}(\mathfrak{L}(A) / \mathfrak{L}(A-T))=0$.

Corollary 1. Let $(V, X)$ be a member of $\Sigma$ and $T=\sum_{i} a_{i} Y_{i}$ the reduced expression for a member of $\Lambda\left(2 c_{5} X\right)$. Set $T^{\prime}=\sum_{i} a_{i}^{\prime} Y_{i}, U=\sum_{i} a^{\prime \prime}{ }_{i} Y_{i}$ with $0 \leqq a_{i}^{\prime}, a^{\prime \prime}{ }_{i} \leqq a_{i}$. Then, there is a constant $c_{10}$ such that

$$
H^{1}\left(\mathfrak{L}\left(2 m c_{5} X-U\right) / \mathfrak{Q}\left(2 m c_{5} X-U-T^{\prime}\right)\right)=0
$$

for $m \geqq c_{10}$.

Proof. This follows at once from our lemma, (I) and from Lemma 2.

Corollary 2. There is a constant $c_{11}$ such that

$$
c_{11} \geqq s\left(2 m c_{5} X\right) \geqq s\left(2(m+1) c_{5} X-B\right) \geqq s\left(2(m+1) c_{5} X\right)
$$

whenever $(V, X) \in \Sigma, l\left(2 c_{5} X-B\right) \geqq 1$ and $m \geqq c_{10}$.

Proof. This is an easy consequence of Theorem 3, Corollary 1 above and of Lemma 2. (cf. Remark 2.)

Corollary 3. There is a constant $c_{12}$ such that $\Lambda\left(2 m c_{5} X\right)$ is irreducible (i.e. contains an irreducible curve) whenever $(V, X) \in \Sigma$ and $m>c_{12}$. 
Proof. Let $T$ be a member of $\Lambda\left(2 c_{5} X\right)$. If $Y$ is a fixed component of $\Lambda(m T)$, we have $l(m T)=l(m T-Y)$. Then $m[T \cdot Y]=s(m T-Y)$ $-s(m T)+p_{a}(Y)-1$ by the theorem of Riemann-Roch, which leads to a ('ontradiction if $m>\max \left(c_{10}, 2 c_{11}+c_{8}-1\right)=c_{11}$ ' by the above Corollary 1 , (I) and by (iii) of Lemma 2. If $\Lambda(m T)$ is composed of a pencil for $m>c_{11}{ }^{\prime}$, a generic divisor of $\Lambda(m T)$ can be written as $\sum_{1}^{t} T_{i}$, where the $T_{i}$ belong to one and the same pencil by the theorem of Bertini. Clearly, we have $\operatorname{dim} \Lambda(m T) \leqq t$ and $\left[T_{i} \cdot T_{j}\right] \geqq 1$ by $(\mathrm{I})$. Hence $(m T)^{(2)} \geqq t^{2}$ and $2 m c_{5} \cdot c_{1}^{\frac{1}{2}} \geqq t \geqq \operatorname{dim} \Lambda(m T)$. On the other hand,

$$
\operatorname{dim} \Lambda(m T) \leqq\left(2 m^{2} c_{5}^{2} c_{1}-m c_{5} c_{4}\right)-c_{3}
$$

by (I), Lemma 1 and by the theorem of Riemann-Roch. Our corollary now follows from this easily.

2. When $\Lambda$ and $\Lambda^{\prime}$ are two linear systems on a complete normal variety, the smallest linear system $\Lambda^{\prime \prime}$ containing the divisors $X+X^{\prime}, X \in \Lambda, X^{\prime} \in \Lambda^{\prime}$, is called the minimum sum of $\Lambda$ and $\Lambda^{\prime}$. Then the following lemma is easy to prove.

Lemma 5. Let $\Lambda(C)$ be a non-empty complete linear system on a complete normal variety $W$. Assume that $\Lambda(C)$ has no base point and that the minimum sum of $\Lambda(C)$ and $\Lambda(m C)$ is complete. Let $h_{i}$ be a nondegenerate map of $W$ into a projective space determined by $\Lambda(i C)$ for $i=m, m+1$. Then there is an isomorphism $\alpha$ between images $W_{m}, W_{m+1}$ of $W$ by $h_{m}, h_{m+1}$ such that $h_{m+1}=\alpha \circ h_{m}$.

In the following three lemmas, denote by $C$ an irreducible curve on a non-singular projective surface $V$ and $\Re_{C}$ the intersection of local rings of $C$ at the singular points of $C$. Using only those functions of $C$ which are in $\Re_{C}$, we can define linear systems as in the case of normal varieties. Throughout this chapter, linear systems on curves lying on $V$ are understood in this sense. By the degree of a linear system on $C$, we understand the degree of a generic divisor of the linear system. The Riemann-Roch theorem on $C$ then states $l(\mathfrak{m})=\operatorname{deg}(\mathfrak{m})-p_{a}(C)+1+l(K(C)-\mathfrak{m})$ for a $C$-divisor $\mathfrak{m}$ (cf. [13], [16]). The following lemma is known as a lemma of Castelnuovo when $C$ is non-singular, which can be proved in the same way as in the ordinary case.

Lemma 6. Let $\Lambda^{\prime}$ be a linear system on $C$ without base point and $\Lambda$ a complete non-special linear system on $C$. Let $\mathfrak{n}^{\prime}$ be a generic divisor of $\Lambda^{\prime}$ and assume that $\Lambda-\mathfrak{n}^{\prime}$ is non-special and is of degree equal to $\operatorname{deg}(\Lambda)$ - $\operatorname{deg}\left(\mathfrak{n}^{\prime}\right)$. Then the minimum sum of $\Lambda$ and $\Lambda^{\prime}$ is complete. 
Actually, it is enough to know a special case of this lemma, under an additional assumption that $\operatorname{deg}(\boldsymbol{\Lambda})-\operatorname{deg}\left(\mathfrak{n}^{\prime}\right) \geqq 2 p_{a}(C)$, which makes a proof very easy.

Let $\Lambda$ be a linear system $\Lambda(M)+F$, where $M$ is a finitely generated module of functions on $V$. Let $C^{*}$ be the largest non-singular open subset of $C$ and denote by * the restriction of a $C$-chain (i.e. a zero-cycle on $V$ whose support is contained in $C$ ) to $C^{*}$. Assume that $C$ and $F$ intersect properly on $V$ and that every $g$ in $I$ induces a function $g^{\prime}$ in $\mathfrak{A}_{C}$ on $C$. Denote by $M I^{\prime}$ the set of such functions $g^{\prime}$ and by $\Lambda^{\prime}$ the set of $C$-divisors $\left(X^{*} \cdot C\right)^{*}+(C \cdot F)^{*}$, by taking for $X$ all divisors from $\Lambda(M)$ such that $X$ and $C$ intersect properly on $T^{\prime}$. Then $\Lambda^{\prime}$ is a linear system on $C$, whose reduced part is determined by $M^{\prime}$. We denote $\Lambda^{\prime}$ by $\operatorname{Tr}_{C} \Lambda$ and call it the linear system on $C$ induced by $\Lambda$. When $\Lambda$ is a complete linear system $\Lambda(X)$ whose fixed component $F$ satisfies our requirement, it always induces a linear system on $C$, since there is a $V$-divisor $Z$ such that $I \sim Z$ and that the support of $Z$ does not contain $C$ and the singular points of $C$.

IAEMIMA \%. Assume that $C^{(2)}>0$ and that $\Lambda(C)$ has no base point. If $s\left(m\left(C^{\prime}\right)\right.$ is a constant for all positive integers $m$, the minimum sum of $\Lambda(C)$ and $\mathrm{\Lambda}(\mathrm{mC})$ is complete for $m>[C \cdot K(V)]+4$.

Proof. Let $C^{\prime}$ be a $V$-divisor such that $C^{\prime} \sim C$ and that $\left|C^{\prime}\right|$ contains neither $C$ nor the singular points of $C$. We have $[C \cdot(m C)]-2 p_{a}(C)>0$ when $m$ satisfies our condition. Hence $\operatorname{Tr}_{C} \Lambda\left(m C^{\prime}\right)$ is complete by Corollary of Lemma 3. By our assumption, $\operatorname{Tr}_{C} \Lambda\left(C^{\prime}\right)$ has no base point. Hence the minimum sum of $\operatorname{Tr}_{C} \Lambda\left(m C^{\prime}\right)$ and $\operatorname{Tr}_{C} \Lambda\left(C^{\prime}\right)$ is complete for such $m$ by Lemma 6. Thus, the minimum sum of $\Lambda\left(m C^{\prime}\right)$ and $\Lambda\left(C^{\prime}\right)$ induces on $C$ a complete linear system. Let $M$ be the module generated by $f \cdot g$ with $f \in L\left(m C^{\prime}\right), g \in L\left(C^{\prime}\right)$. Then $M$ induces on $C$ the module $I .\left((m+1) C^{\prime} \cdot C\right)$. When $h$ is a function in $L\left((m+1) C^{\prime}\right)$, inducing 0 on $C$, we have $\operatorname{div}(h)$ $=C+H-(m+1) C^{\prime}$ with $H>0$. Hence $h$ is in $M$ (cf. [18], Chap. IX, ('or. 2 of Th. 8). Our lemma follows from this at once.

It is not true in general that a complete linear system $\Lambda(\mathrm{m})$ on $C$ contains a divisor of the same degree as $\mathfrak{m}$, unless $\mathfrak{n}$ is positive. But we have the following.

LEMMA 8. When $\operatorname{deg}(\mathfrak{m}) \geqq 2 p_{a}(C), \Lambda(\mathfrak{n l})$ contains a divisor of the same degree as m.

Proof. Let $\mathfrak{a}$ be a positive $C$-divisor sur h that $\operatorname{deg}(\mathfrak{m})=\operatorname{deg}(\mathfrak{a})=m$. Set $p_{a}(C)=t$ and let $k$ be a field of definition for $C$ over which $\mathfrak{m}$ and $a$ 
are rational. Then, there are two generic divisors $\mathfrak{p}$ and $\mathfrak{q}$ of degrees $t$ orer $k$ and a unit $f$ in $\Re_{C}$ such that $\operatorname{div}(f)=(\mathfrak{m}-\mathfrak{a})+(\mathfrak{p}-\mathfrak{q})$ (cf. [14], Lemma 3). Since $\mathfrak{a}$ is positive, a generic divisor of $\Lambda(\mathfrak{a})$ over $k$ has the degree $m$; moreover, we have $l(\mathfrak{a}) \geqq t+1$. Therefore, there is a unit $g$ in $\mathfrak{R}_{C}$ and a generic divisor $\mathfrak{a}^{\prime}=\mathfrak{p}+\mathfrak{b}$ of $\Lambda(\mathfrak{a})$ of degree $m$ such that $\operatorname{div}(g)$ $=\mathfrak{a}-\mathfrak{a}^{\prime}$. Then $\operatorname{div}(f \cdot g)=\mathfrak{m}-\mathfrak{q}-\mathfrak{b}$ and our lemma is proved.

3. THEOREM 4. There is a constant $c_{13}$ such that m.Y is ample for all $(V, X) \in \Sigma$, whenever $m>c_{13}$.

Proof. In order to prove our theorem, it is enough to prove that $m_{0} X$ is ample for all $(V, X)$ in $\mathbf{\Sigma}$, where $m_{0}$ is a constant, depending only on $c_{1}$, $c_{2}, c_{3}$. In fact, the sum of two ample divisors is also ample; moreover, if $Y$ is an ample divisor on a non-singular projective variety $W$ and $B=B^{\prime}-B^{\prime \prime}$, $B^{\prime} \succ 0, B^{\prime \prime}>0$, is a $W$-divisor, then, whenever

$$
d \geqq \operatorname{deg}\left(B^{\prime}\right) \cdot(\operatorname{deg}(W)-2)+\operatorname{deg}(V)+\operatorname{deg}\left(B^{\prime \prime}\right),
$$

$d Y+B$ is ample (cf. [18], Chap. IX, Cor., Th. 13). Our theorem follows from these two facts and from Lemma 1 as an easy exercise.

Let $T$ be a member of $\Lambda\left(2 c_{5} X\right)$ and $Z$ an irreducible member of $\Lambda\left(c_{12} T\right)$ (cf. Cor. 3 of Lemma 4). Then we have

(a) $[r Z \cdot(2 t Z+i T)]>2 p_{a}(r Z)$ for $0<r \leqq t$ and for all $i \geqq 0$. In fact, this follows from (II), Lemma 1 and from the formula $2 p_{a}(D)-2$ $=[D \cdot(D+K(V))]$.

(b) There is a constant $d$, depending only on $c_{1}, c_{2}, c_{3}$, such that $\Lambda(m d Z)$ has no base point for $m \geqq 1$. In fact, there is an integer $a$ such that $3 \leqq a \leqq c_{11}+3$ and that $s(a Z-Z)=s(a Z)$ (cf. Cor. 2 of Temma 4 ). Set $r=t=1$ in (a). Then we see that $\operatorname{Tr}_{Z} \Lambda(a Z)$ is complete by Corollary of Lemma 3 ; moreover, it is of degree $>2 p_{a}(Z)$ since $\mathbb{R}(a Z) / \mathfrak{R}(a Z-Z)$ is isomorphic to $\mathfrak{L}((a Z) \wedge Z)$. When that is so, $\Lambda(a Z)$ has no base point by Lemma 8.

Now let $E$ be an irreducible member of $\Lambda(d Z)$ (cf. Cor. 3 of Lemma 4 ). By Corollary 2 of Lemma 4 , there is an integer $b$ such that $3 \leqq z \leqq c_{11}+3$ and that $s(b E)=s(b E-E)$. $\operatorname{Tr}_{E} \Lambda(E)$ has no base point by (b). By (a) and Corollary of Lemma $3, \operatorname{Tr}_{E} \Lambda(b E)$ is complete and is of degree $>2 p_{a}(E)$. Moreover, $\operatorname{Tr}_{E} \Lambda(E)$ and $\operatorname{Tr}_{E} \Lambda(b E)$ satisfy the conditions of Lemma 6 by (a) and (b). Therefore, the minimum sum of them is complete, which implies that $\operatorname{Tr}_{E} \Delta(b E+E)$ is complete and $s(b E+E)=s(b E)$ 
(cf. Cor. of Lemma 3). Repeating this, we see that $s\left(c_{11} E+3 E+m E\right.$ ) is a constant for all $m \geqq 1$. By (I) and (b), a non-degenerate map $h_{m}$ of $V$ into a projective space, determined by $\Lambda\left(m\left(c_{11}+3\right) E\right)$, is a morphism and has no fundamental curve on $V$. Then it is a projective embedding for large $m$, which is an easy consequence of the possibility of projective normalization in an algebraic extension of the function field (cf. [7], Chap. IV, Prop. 8). Then $h_{m}$ is already a projective embedding if

$$
\left.m>\left[c_{11}+3\right) E \cdot K(V)\right]+r \geqq 2\left(c_{11}+3\right) d c_{12} c_{5} c_{4}+4
$$

by Lemma 5 and Lemma $\%$. Our theorem is thereby proved.

\section{Chapter IV.}

So far, we have restricted our technique to the use of irreducible curves on the surface. Since the generalized Riemann-Roch theorem is available for such curves, it was easy to see, for instance, whether some linear systems on such curves are free from base points, etc. On the other hand, if we generalize a criterion of ampleness to reducible curves by means of the theory of schemes, we can simplify the latter part of Chapter III to some extent. Let $V$ be a projective variety. Denote by $\mathfrak{D}_{V}$ the sheaf of local rings on $V$. If $D$ is a Cartier divisor on $V$, we mean by the associated subscheme $\mathscr{D}$ the subscheme of the scheme $V$ (a) whose underlying space is the support of $D$ and (b) whose sheaf $\mathfrak{D}_{\mathscr{D}}$ is defined at a point $x$ of $D$ to be $\mathfrak{D}_{x, V} /(f)$ for any local equation $f$ of the divisor $D$, where $\mathfrak{D}_{x, V}$ is the local ring of $V$ at $x$. We further denote by $\mathfrak{m}_{x, \mathfrak{v}}$ the maximal ideal of $\mathfrak{D}_{x, \mathfrak{r}}$. In the following proposition, we discuss a criterion of ampleness on a positive 1-cycle on $V$. The first half of the proposition has been settled essentially in Lemma 4, and we give only a brief account of the proof for it in the sheaf theoretic terminology.

Propositiox. Let $T=\sum_{1}^{t} a_{i} Y_{i}$ be a positive divisor on a non-singular projeclive surface $W$. Let $\tau$ be the subscheme of the scheme $W$, associated to $T$, and $\mathfrak{M}$ an invertible sheaf on $\tau$. Let $d_{i}$ be the degree of the Cartier. divisor class on $Y_{i}$ defined by $\mathfrak{M} \otimes \mathfrak{D}_{Y_{i}}$. If $d_{i}>\left[\left(K(W)+T^{\prime}\right) \cdot Y_{i}\right]$ for all .divisors $T^{\prime}$ such that $0 \prec T^{\prime} \prec T$, and for all $i$ such that $1 \leqq i \leqq t$, it follows that $H^{1}(\mathfrak{M})=0$. Moreover, if $d_{i}>\left[\left(K(W)+T^{\prime}\right) \cdot Y_{i}\right]+2 \max _{x \in Y_{i}}$ (multiplicity of $x$ on $\left.Y_{i}\right)$, then $\mathfrak{M}$ is ample on $\tau$.

Proof. We have $\mathfrak{D}_{W} / \mathfrak{I}(-T) \cong \mathfrak{D}_{\tau}, \mathfrak{D}_{W} / \mathbb{R}\left(-T^{\prime}\right) \cong \mathfrak{D}_{\tau^{\prime}}$ from the definitions of $\mathfrak{D}_{\tau}, \mathfrak{D}_{\tau^{\prime}}$, where $\tau^{\prime}$ is the subscheme of $W$, associated to $T^{\prime}$. More- 
over, we have $\mathfrak{L}\left(-T^{\prime}\right) / \mathfrak{L}(-T)=\mathfrak{L}\left(-T^{\prime}\right) \otimes \mathfrak{D}_{Y_{1}}$ when we use the same notations as in the proof of Lemma 4 . Hence, we have the exact sequence:

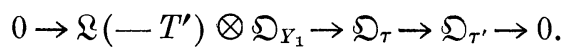

Tensoring the above with $\mathfrak{M}$, we get

$$
H^{1}\left(\mathfrak{M} \otimes \mathfrak{L}\left(-T^{\prime}\right) \otimes \mathfrak{S}_{Y_{1}}\right) \rightarrow H^{1}(\mathfrak{M}) \rightarrow H^{1}\left(\mathfrak{M} \otimes \mathfrak{\sim}_{\tau^{\prime}}\right) \rightarrow 0
$$

instead of

$$
\begin{gathered}
H^{1}\left(\mathfrak{\sim}\left(A-T^{\prime}\right) / \mathfrak{\sim}(A-T)\right) \rightarrow H^{1}(\mathfrak{L}(A) / \mathfrak{Q}(A-T)) \rightarrow \\
\rightarrow H^{1}\left(\mathfrak{L}(A) / \mathfrak{R}\left(A-T^{\prime}\right)\right) \rightarrow 0
\end{gathered}
$$

in the proof of Lemma 4. Computing the degree of the Cartier divisor class on $Y_{1}$ determined by $\mathfrak{M} \otimes \mathfrak{L}\left(-T^{\prime}\right) \otimes \mathfrak{D}_{Y_{1}}$, we get $H^{1}(\mathfrak{M})=0$ as in the proof of Lemma 4 .

For an invertible sheaf $\mathfrak{R}$ on a complete algebraic scheme $W$ to be ample, it is necessary and sufficient that:

(i) the sections of $\mathfrak{a}$ separate points,

(*) (ii) for any point $x$ on $\mathrm{W}$, if we identify the stalk $\mathfrak{\Omega}_{x}$ with $\mathfrak{\Omega}_{x, \mathrm{~W}}$, the sections of $\mathbb{\Omega}$ which are zero at $x$ span $\mathfrak{m}_{x, W} / \mathfrak{m}_{x, w^{2}}$.

For every point $x$ in the support $|T|$ of $T$, let $D_{x}$ be a positive $W$-divisor such that $i\left(D_{x} \cdot Y_{i}, x ; V\right)$ gives the multiplicity of $x$ on $Y_{i}$ for $1 \leqq i \leqq t$. Moreover, for every pair of distinct points $x, y$ in $|T|$, define an invertible sheaf $\mathfrak{M}_{x, y}$ on $\tau$ as follows:

$$
\begin{aligned}
\mathfrak{M}_{x, y} & =\mathfrak{M} \otimes \mathfrak{L}\left(-D_{x}\right) \text { in }|T|-y-\left(|T| \cap\left|D_{x}\right|-x\right), \\
& =\mathfrak{M} \otimes \mathfrak{Q}\left(-D_{y}\right) \text { in }|T|-x-\left(|T| \cap\left|D_{y}\right|-y\right), \\
& =\mathfrak{M} \text { elsewhere. }
\end{aligned}
$$

Similarly, for every $x$ in $|T|$, define $\mathfrak{M}_{x, x}$ as follows:

$$
\begin{aligned}
\mathfrak{M}_{x, x} & =\mathfrak{M} \otimes \mathfrak{L}\left(-2 D_{x}\right) \text { in }|T|-\left(|T| \cap\left|D_{x}\right|-x\right), \\
& =\mathfrak{M} \text { elsewhere. }
\end{aligned}
$$

The first half of the proposition implies $H^{1}\left(\mathfrak{M}_{x, y}\right)=0$ for all the pairs $(x, y)$, because the degree of the restriction of $\mathfrak{M}_{x, y}$ to $Y_{i}$ is at least $d_{i}-2 \max _{z \in Y_{i}}$ (multiplicity of $z$ on $Y_{i}$ ). Therefore, we have the exact sequence

$$
0 \rightarrow H^{0}\left(\mathfrak{M}_{x, y}\right) \rightarrow H^{0}(\mathfrak{M}) \rightarrow H^{0}\left(\mathfrak{M} / \mathfrak{M}_{x, y}\right) \rightarrow H^{1}\left(\mathfrak{M}_{x, y}\right)=0
$$


for all pairs $(x, y)$. Now suppose $x \neq y$. Then $H^{0}\left(\mathfrak{M} / \mathfrak{M}_{x, y}\right)$, whose support is the union of two points $x$ and $y$, contains a section which is 0 at $x$ and a unit at $y$. Hence $H^{0}(\mathfrak{M})$ contains a section with the same property. Then the sections of $\mathfrak{M}$ separates points. Let $f=0$ be a local equation of $D_{x}$ at $x, f$ being an element of $\mathfrak{D}_{x, W}$. Then $f$ induces an element $f^{\prime}$ in $\mathfrak{D}_{x, \tau}$, and we have $\left(\mathfrak{M} / \mathfrak{M}_{x, x}\right)_{x}=\mathfrak{D}_{x, \tau} / f^{\prime 2}$. But this maps surjectively to the ring $\mathfrak{D}_{x, \tau} / \mathfrak{m}_{x, \tau}{ }^{2}$. Hence, if we identify $\mathfrak{M}_{x}$ to $\mathfrak{D}_{x, \tau}$, the sections of $\mathfrak{M}$ which are zero at $x \operatorname{span} \mathfrak{m}_{x, \tau} / \mathfrak{m}_{x}, \tau^{2}$.

Using this proposition, we recover Corollary 1 and Corollary 2 of Lemma 4. Moreover, Corollary 1 can be expressed as $H^{1}\left(\mathfrak{L}(m X-U) \otimes \mathfrak{D}_{\tau}\right)$ $=0$ for $m \geqq c_{10}$, where $\tau$ is the subscheme of $V$, corresponding to a member $T$ of $\Lambda\left(2 c_{,} X\right)$. Furthermore, we see that $\mathfrak{L}(m X) \otimes \mathfrak{D}_{\tau}$ is ample on $\tau$ for $m \geqq c_{10}$ when $c_{10}$ is chosen suitably. Then, by Corollary 2 of Lemma 4, one can find an integer $r$ such that $s\left(c_{10} T+r T\right)=s\left(c_{10} T+r T+T\right)$ and that $0 \leqq r \leqq c_{11}$. Set $m=2 c_{5}\left(c_{10}+r+1\right)$. Since $H^{1}\left(\mathfrak{L}(m X) \otimes \mathfrak{D}_{\tau}\right)=(0)$ and $s(m X)=s(m X-T)$, it follows that the restriction map $H^{0}(\mathbb{L}(m X))$ $\rightarrow H^{0}\left(\Omega(m X) \otimes \mathfrak{D}_{\tau}\right)$ is surjective. Moreover, $\mathfrak{L}(m X) \otimes \mathfrak{D}_{\tau}$ is ample on $\tau$. To prove that $\mathfrak{\Omega}(m X)$ is ample, we again use the criterion (*) cited above. Let $x$ and $y$ be two given points on $V$. Let $T_{1}$ and $T_{2}$ be members of $\Lambda\left(c_{5} X\right)$ such that $T_{1}$ goes through $x$ and that $T_{2}$ goes through $y$ (cf. Lemma 1 ). When we set $T=T_{1}+T_{2}, x$ and $y$ are in the support of $\tau$. Since $\mathfrak{L}(m X) \otimes \mathfrak{D}_{\tau}$ is ample, there is a section of this sheaf which is zero at $x$ and not zero at $y$ if $x \neq y$. Lifting this to a section of $\mathfrak{L}(m X)$, the same is true of $\mathfrak{L}(m X)$. If $x=y$, set $T=2 T_{1}$. The restriction of functions from $V$ to $\tau$ induces an isomorphism between $\mathfrak{m}_{x, v} / \mathfrak{m}_{x, v^{2}}$ and $\mathfrak{m}_{x, \tau} / \mathfrak{m}_{x, \tau}{ }^{2}$. When we identify the stalk of $\mathfrak{L}(m X) \otimes \mathfrak{D}_{\tau}$ at $x$ with $\mathfrak{D}_{x, \tau}$, the sections of $\mathfrak{L}(m X) \otimes \mathfrak{D}_{\tau}$ which vanish at $x$ span $\mathfrak{m}_{x, \tau} / \mathfrak{m}_{x, \tau}{ }^{2}$. Lifting these sections, we see that the same is true of $\mathfrak{L}(m X)$ and $\mathfrak{m}_{x, v} / \mathfrak{m}_{x, v^{2}}$. Hence $\mathfrak{\Omega}(m X)$ is ample for $m=2 c_{5}\left(c_{1}+r+1\right)$, where $r$ is a certain integer such that $0 \leqq r \leqq c_{11}$. Since the sum of two ample divisors is ample, we see that $\mathfrak{\Omega}(d X)$ is ample for a suitable $d$, which depends only on $c_{1}, c_{2}, c_{3}$. Then we get our Theorem 4 again. 


\section{REFERENCES.}

[1] S. Abhyankar, "On the valuations centered in a local domain," American Journal of Mathematics, vol. 78 (1956), pp. 321-348.

[2] A. Grothendieck, "Eléments de géometrie algébriques, I, II, III," Publications Mathematiques Institut des Hautes Etudes Scientifiques, Paris.

[3] H. Hironaka, "A note on algebraic geometry over ground rings," Illinois Journal of Mathematics, vol. 2 (1958), pp. 355-366.

[4] - " "On the arithmetic genera and the effective genera of algebraic curves," Memoirs of the College of Science, University of Kyoto, vol. 30 (1957), pp. 177-195.

[5] K. Kodaira and D. C. Spencer, "On deformations of complex analytic structures, I, II," Annals of Mathematics, vol. 67 (1958), pp. 328-466.

[6] M. Kuranishi, "On the locally complete families of complex analytic structures," Annals of Mathematics, vol. 75 (1962), pp. 536-577.

[7] S. Lang, Introduction to Algebraic Geometry, Interscience Tracts, No. 5, 1958.

[8] T. Matsusaka, "Polarized varieties, fields of moduli and generalized Kummer varieties of polarized Abelian varieties," American Journal of Mathematics, vol. 80 (1958), pp. 45-82.

[9] — "Q-varieties," to appear.

[10] D. Mumford, "Geometric invariant theory," to appear.

[11] — " "Further pathologies in algebraic geometry," American Journal of Mathematics, vol. 84 (1962), pp. 642-647.

[12] Y. Nakai, "Non-degenerate divisors on an algebraic surface," Joumal of Science of the Hiroshima University, vol. 24 (1960), pp. 1-6.

[13] M. Rosenlicht, "Equivalence relations on algebraic curves," Annals of Mathematics, vol. 56 (1952), pp. 169-191.

[14] —_, "Generalized Jacobian varieties," Annals of Mathematics, vol. 59 (1954), pp. 505-530.

[15] J.-P. Serre, "Faisceaux algébriques coherents," Annals of Mathematics, vol. 61 (1955), pp. 197-278.

[16] —-, Groupes algébriques et corps de classes, Actualités Scientifiques et Industrielles, No. 1264, Paris.

[17] G. Shimura, "Reduction of algebraic varieties with respect to a discrete valuation of the basic field," American Journal of Mathematics, vol. 77 (1955), pp. 134-176.

[18] A. Weil, Foundations of algebraic geometry, revised edition, American Mathematical Society Colloquium Publications, No. 29 (1960).

[19] - Variétiés Abéliennes et Courbes algébriques, Actualités Scientifiques et Industrielles, No. 1064, Paris.

[20] - "On the theory of complex multiplications," Proceedings, International Symposium on Algebraic Number Theory, Nikko, Japan, 1955.

[21] O. Zariski, "Complete linear systems on normal varieties and a generalization of a lemma of Enriques-Severi," Annals of Mathematics, vol. 55 (1952), pp. $552-592$.

[22] - "Scientific report on the second summer institute, III," Bullctin of the American Mathematical Society, vol. 62 (1956), pp. 117-141. 